\title{
LA GESTIÓN DEL PRESUPUESTO POR RESULTADOS Y LA CALIDAD DEL GASTO EN GOBIERNOS LOCALES
}

\section{THE MANAGEMENT OF THE BUDGET FOR RESULTS AND THE QUALITY OF EXPENDITURE IN LOCAL GOVERNMENTS}

\author{
Jorge Alberto Vargas Merino \\ Universidad Norbert Wiener, Facultad de Ingeniería y \\ Negocios, Lima, Perú \\ jorgealbvarmer5@gmail.com
}

\author{
Walter Enrique Zavaleta Chávez \\ Universidad César Vallejo, Facultad de Ciencias \\ Empresariales, Lima, Perú \\ wzavaletac@ucv.edu.pe
}

Fecha de recepción: 10/03/2020 - Fecha de aprobación: 04/05/2020
DOI: https://doi.org/10.36995/j.visiondefuturo.2020.24.02.002.es

\section{RESUMEN}

Esta investigación tuvo un diseño no experimental, longitudinal y correlacional predictivo. Se trabajó con los 83 gobiernos locales de la Región La Libertad en Perú, y utilizó el análisis de Contenido como técnica de investigación. El diagnóstico de la gestión del presupuesto por Resultados arroja un nivel de ejecución de un $84 \%$ a nivel de La Región en estudio en comparación con la ejecución alcanzada por los 83 distritos cuya ejecución alcanza sólo un $66 \%$. Se midió la calidad del gasto, identificando los indicadores que guían el mejoramiento de los niveles de vida de la población, en salud y educación principalmente, observándose mejoras significativas, aunque aún existe una brecha importante. Se confirma que existe una relación directa y significativa entre la gestión del presupuesto por resultados y la calidad del gasto; lo cual se comprueba en primer lugar con el nivel de significancia global del modelo estadístico [Prob (F Statistic) = 0.0000], y además con el coeficiente de correlación de 0.69, principalmente con los indicadores de programas de Saneamiento Urbano y Rural, Programa de Acceso y Uso adecuado de Servicios de Telecomunicaciones, y finalmente, el Programa de Logros de Aprendizaje de EBR (Educación Básica Regular).

PALABRAS CLAVE: Presupuesto por Resultados; Programas Presupuestales; Gestión; Calidad de Vida; Gasto Público.

\section{ABSTRACT}

This research had a non-experimental, longitudinal and correlational predictive design.

\footnotetext{
"Visión de Futuro" Año 17, Volumen N² 24 N², Julio - Diciembre 2020 - Pág 37 - 59 URL de la Revista: http://visiondefuturo.fce.unam.edu.ar/index.php/visiondefuturo/index URL del Documento: https://visiondefuturo.fce.unam.edu.ar/index.php/visiondefuturo/issue/view/18 ISSN 1668 - 8708 - Versión en Línea 
We worked with the 83 local governments of the La Libertad Region in Peru, and used content analysis as a research technique. The diagnosis of budget management by results shows an execution level of $84 \%$ at the level of The Region under study compared to the execution reached by the 83 districts whose execution reaches only $66 \%$. The quality of expenditure was measured, identifying the indicators that guide the improvement of the population's living standards, mainly in health and education, and observing significant improvements, although there is still a significant gap. It is confirmed that there is a direct and significant relationship between results-based budget management and the quality of spending; this is verified firstly with the level of global significance of the statistical model [Prob (F Statistic) $=0.0000$ ], and also with the correlation coefficient of 0.69 , mainly with the indicators of the Urban and Rural Sanitation programs, the Access and Adequate Use of Telecommunications Services Program, and finally, the RBS (Regular Basic Education) Learning Achievement Program.

KEY WORDS: Budget for Results; Budgetary Programmes; Management; Quality of Life; Public Expenditure.

\section{INTRODUCCIÓN}

Actualmente existe una clara concordancia de los diversos actores de la sociedad, de que la Administración Pública es prestadora de gran insatisfacción, por su actuar definido por servicios o procesos lentos, caros, corruptos, de baja o mala calidad; que además de estar mal diseñados, ya que no se centran, ni mucho menos cubren la necesidades más apremiantes de la población, sino que también, como si no bastara con ello, se producen despilfarros de recursos humanos y financieros, que indignan a todos.

Según Schröder, (2013), aunado a ello, se debate el denominado buen gobierno, que lo que busca en primer lugar es entender cuáles constituyen las fuentes de los defectos que existen y que degeneran en una administración pública ineficiente, corrupta, derrochadora de recursos, que utiliza los compadrazgos por encima de la idoneidad para el cabal cumplimiento de la función pública. Por tanto, lo que buscará el buen gobierno es una representación de gobierno y de administración pública que sea competente para proveer la naturaleza de sí misma de forma eficiente y eficaz, es decir, lograr el fin último, la satisfacción de las necesidades de la población.

Definiendo lo que significa el buen gobierno, podemos llegar a comprender las exigencias de la Nueva Gestión Pública (en adelante NGP). Según Schröder, P (2013), en resumen la NGP posee las siguientes características; una dirección que se orienta a la competencia, lográndose ello, a través de la separación de las competencias entre los que financian y prestan el servicio; un enfoque en la efectividad, es decir la suma de eficiencia y 
eficacia en el cumplimiento de las tareas; una disociación de la dirección estratégica de la dirección operativa; trato igualitario entre prestadores de servicios particulares y públicos como parte de un marco de prestación de servicios y presupuestos globales; y por último un enfoque de innovación por una gerencia que no solo es descentralizada sino que es capaz de delegar idóneamente.

En el marco de este nuevo enfoque, Franciskovic, (2013) refiere que los mecanismos para concretar el presupuesto público y aquellos procesos orientados a su seguimiento y evaluación se convierten en elementos claves para garantizar la eficiencia y transparencia de la función pública. Además, Franciskovic (2013) a su vez hace hincapié que los aspectos clave que deben orientar la elaboración presupuestal son, entre otros, la inclusión de espacios de participación, la definición clara y precisa de conceptos, procedimientos, instrumentos y responsabilidades. Por otra parte, refiriéndose a la rendición de cuentas como el efecto preventivo de actividades ilegales y corruptas, lo que significa lograr responsabilizar a los funcionarios por sus actos; es decir, de lo que se trata, es de alcanzar un manejo honesto de los fondos públicos, pero además de ello, y lo más importante, es ser responsables por el cumplimiento de metas y adecuación de las políticas a las necesidades de la población.

Es así que en el 2007, el Perú se dispuso, enmarcándose dentro de la NGP, a implementar la gestión por resultados, que viene a ser un modelo de administración de los recursos públicos centrado en el cumplimiento de las acciones estratégicas del plan de gobierno para un determinado período de tiempo y con determinados recursos; se trata de implementar un enfoque de elaboración presupuestal por resultados, el denominado Presupuesto por Resultados, que según el Ministerio de Economía y Finanzas (en adelante $\mathrm{MEF}$ ), consiste en la aplicación de principios y técnicas para el diseño (Programas Presupuestales), ejecución, seguimiento y evaluación del presupuesto relacionando bienes y servicios (productos) y cambios generados en el bienestar ciudadano (resultados). De lo que se trata finalmente es de reencausar la dirección y misión de cada uno de los organismos públicos hacia un modelo de gestión que priorice los resultados por sobre los procedimientos y en donde predomine la transparencia en la gestión, sólo así se logrará calidad en el gasto y el fin último de bienestar social, que todos queremos.

Dentro del marco de una nueva gestión de la administración pública de y para la calidad, en el Perú desde el 2007, el MEF decidió implementar de manera progresiva el Presupuesto por Resultados (PpR); por ello se hace necesario conocer si esta nueva forma de manejar las finanzas públicas, sirve y servirá para impulsar el desarrollo económico y social del país, y de qué forma concreta podrá contribuir a superar los problemas álgidos que nos agobian.

En la actualidad, una gestión pública realmente efectiva, es aquella que obtiene logros palpables para su sociedad; se trata de sobrepasar los simples insumos o productos, que pueden producir efectos efímeros en el bienestar de los ciudadanos; para llegar a tener 
impactos perdurables en la calidad de vida de la población. Carrasco (2015).

Con respecto a los gobiernos locales, el presupuesto que se les concede no está enfocado en los beneficios que el gasto o la inversión generen en el ciudadano. En los últimos años los gobiernos locales, de todo el país, y los de la Región La Libertad, han visto incrementados sus recursos para atender sus gastos operativos y de inversión, sin embargo, diferentes análisis concluyen que existen muchos problemas sociales que no son atendidos en el Perú.

Del análisis de la información del gasto de los gobiernos locales en La Libertad, se observa la característica incremental del presupuesto tradicional. Al parecer al Ministerio de Economía y Finanzas, a pesar del diseño de una gestión por resultados, sólo le está interesando saber cuánto se gastó, pero no se está haciendo una evaluación efectiva del resultado que se obtiene en la población, no existen indicadores bien diseñados para evaluar el impacto en el gasto social; en los últimos años ha existido un retroceso en este nuevo enfoque en el país.

Los gobiernos locales en el Perú, deben comprometerse a invertir en programas de mejora para los servicios básicos y en salud y nutrición, con esto se logrará mejorar la calidad de vida de la población; las grandes brechas sociales deben ser priorizadas en los presupuestos municipales; y la forma de evaluación no se debe restringir con informar la totalidad de los gastos ejecutados, sino de lo que se trata es de enfocar en cuánto se logra cambiar la calidad de vida de la población, sobre todo de la más vulnerable. Cumplir con las metas presupuestales, gastar en el mejor de los casos todo lo presupuestado, es sinónimo de eficiencia, pero no se atiende a la calidad de dichas metas, no se analiza si la manera en que se ejecuta el gasto público beneficia la mejora de los niveles de calidad de vida de una comunidad.

Según Prieto (2012) el gasto público, debe realizarse con mesura y prudencia, generando un verdadero valor agregado a los servicios públicos; es decir la gestión debe enfocarse a mejorar la calidad de vida de la población, sobre todo de la más pobre. La gestión debe enfocarse hacia una nueva perspectiva centrada en resultados, donde el presupuesto por resultados resulta su herramienta clave, y en nuestro país se viene implementando con cuatro instrumentos, los programas presupuestales, el seguimiento, las evaluaciones independientes y los incentivos a la gestión.

El aporte de la presente investigación radica en estudiar la forma cómo los gobiernos locales utilizan los recursos públicos, para ello se propone analizar el funcionamiento del Presupuesto por Resultados y evaluar la calidad del gasto público que realizan las municipalidades. El objetivo de este modelo presupuestal es mejorar la calidad de la inversión pública y la calidad del gasto. A nivel del gobierno central, servirá para la comprensión de una correcta asignación de los recursos públicos hacia los gobiernos locales, mientras que a los 
gobiernos locales les servirá para tomar decisiones relacionadas al mejoramiento de la calidad de vida de los ciudadanos; si su implementación y aplicación es la óptima, ésta tendrá un impacto muy positivo en los pobladores de las zonas más pobres del País.

Existe una clara necesidad de profundizar en la aplicación del Presupuesto por resultados, para mejorar la efectividad del gasto financiero y presupuestal y lograr mejorar la calidad de vida de la población. Por ello se formula el siguiente problema de investigación, ¿De qué manera se relaciona la gestión del presupuesto por resultados con la calidad del gasto de los gobiernos locales de la Región La Libertad? Se tiene como objetivo, analizar la relación entre la gestión del Presupuesto por Resultados y la calidad del gasto de los gobiernos locales de la Región La Libertad, diagnosticar la gestión del presupuesto por Resultados en los gobiernos locales de la región La Libertad, medir la calidad del gasto, identificando los indicadores de la evaluación presupuestal que guían el mejoramiento de los niveles de vida de la población en los gobiernos locales de la región La Libertad, determinar la relación que existe entre la gestión del presupuesto por Resultados y la Calidad del gasto.

\section{DESARROLLO}

\section{Breve reflexión conceptual}

Armijo \& Espada (2014) manifiestan que en los últimos años se debate el rol de un Estado en el que existe tanto la necesidad de ejecutar actividades para el bienestar social, como la eficiencia en su consecución.

Las investigaciones más recientes relacionadas a medir los efectos del gasto público, pasan por determinar que con éste se busca lograr en primer lugar la estabilización, asignación, y distribución de los recursos, además del rol de las instituciones y las reglas que las rigen; además se habla de la posibilidad de privatización de algunas actividades de la Administración Pública. La conclusión mayoritaria es que el gasto público tendría que ser mucho menor y más eficiente de lo que es hoy en día.

Pero cómo hacer que la Administración Pública sea más eficiente y eficaz. Según Afonso, Schuknecht y Tanzi (2005) citados por Armijo \& Espada (2014) quienes realizan una aproximación para la medición del desempeño del sector público, definen indicadores de desempeño; los denominados de proceso o de oportunidad, que miden los resultados obtenidos en las áreas administrativas, de educación, de salud y de infraestructura pública; y por el otro lado tenemos los indicadores que miden los resultados de la compleja interacción entre el funcionamiento del mercado y los recursos que el gobierno adopta frente a ello.

Para la comprensión de los indicadores de oportunidad, en cuanto al desempeño administrativo, los índices que nos ayudan a comprenderlo son la sumatoria de los índices de corrupción y de formalidades burocráticas, el índice de confianza o calidad de la 
Administración de Justicia y el índice del tamaño de la economía informal.

En cuanto a educación, la medición tanto de la cantidad como de la calidad de la misma, se sigue a través de una serie de parámetros como la prueba Pisa u otros índices de la Organización para la Cooperación y el Desarrollo Económicos (OCDE). Mientras que, en salud, el desempeño pasa por los índices de mortalidad infantil y esperanza de vida, se trata de medir la igualdad de acceso y la calidad de los servicios que se brindan; y en infraestructura pública se mide la calidad de las redes de transportes y comunicaciones, junto con los niveles de cobertura alcanzados.

En el segundo grupo de indicadores destacan la medición de la distribución de ingreso, la estabilidad económica, crecimiento del Producto Bruto Interno (PBI), inflación, desempleo, entre otros identificados en la literatura económica.

Para analizar la calidad del gasto público, se requiere de una mirada amplia, que no quede sólo en un mero análisis de su nivel de composición, por ello Armijo, \& Espada (2014) proponen analizar la calidad del gasto desde una triple función; la primera es el campo macro, es decir hay estabilidad macroeconómica, se logran los objetivos de la política económica; la segunda función es el logro de la eficiencia asignativa, es decir si se gasta para lograr efectividad de los programas públicos priorizados; y la tercera función es la eficiencia operacional, esto significa la capacidad con la que se utilizan los recursos asignados, ya que siendo utilizados oportunamente se alcanzan los resultados.

Dentro del marco de la NGP o la Gestión por Resultados, Raymundo (2011) resalta que se espera que el rol presupuestal, sea un rol más activo; ya que el gasto público debe estar necesariamente vinculado a los objetivos de desarrollo que están priorizados en los planes de gobierno; y los mecanismos que ayudan a éste propósito pasan por una correcta definición de objetivos estratégicos que se concreticen en resultados e impactos esperados de largo alcance y a largo plazo; la producción pública y los resultados son consecuencia de estructuras programáticas presupuestarias coherentes; y por último un mecanismo imprescindible es la información de desempeño, es decir monitorear y evaluar acertadamente las políticas y los programas.

Dentro de este nuevo enfoque, según Tanaka (2011), la información del desempeño resulta clave, ya que además de conectar la planificación y la acción, se logra la eficiencia y la calidad del gasto, lo que se vislumbra en resultados perdurables. Pero esta información de desempeño tiene que ser generada y apoyada por sistemas que no dejen espacio al error, en todas sus fases, desde la formulación, aprobación, ejecución y evaluación o auditoría.

Para implementar el Presupuesto por Resultados, se tiene que cambiar la metodología tradicional, a una metodología en donde los bienes y servicios o productos que las entidades brindan a la colectividad, cuentan con mayor participación dentro de las áreas que las engendran o producen. Para Ausejo, (2010) el Presupuesto por Resultados debe contemplar 
una serie de principios y técnicas para diseñar, ejecutar, hacer seguimiento y evaluar, el conjunto de intervenciones que tienen dos lineamientos claros; primeros los bienes y servicios que se brindan a la población objetivo, que son los productos; y segundo los cambios que se generarían en el bienestar que son los resultados, y que tienen que concretarse con eficiencia, eficacia y equidad.

Robinson \& Last (2009) señalan que, en el Presupuesto por Resultados, las personas tomadoras de decisiones, deben tomar en cuenta metódicamente los resultados, que son respaldados con un gasto coherente. Por ello resulta clave contar con información tanto de objetivos como de resultados, se trata pues de evaluar los programas con indicadores de desempeño de una forma simple.

Para concretar, todo lo antes mencionado, es imprescindible contar con la parte estratégica dentro del manejo del ciclo presupuestario, realizar un proceso de revisión del gasto y con ello comprobar las prioridades del gasto, pero también comprobar si los programas vigentes son adecuados, y más que adecuados, eficientes y eficaces, ya que de este análisis los programas pueden o bien reducirse, o eliminarse, en el peor de los casos, o ampliarse, si la información que nos proporcionan los indicadores así lo demuestran.

Según Carrasco (2015), en el Presupuesto por Resultados la información de resultados en todas las etapas del ciclo presupuestario es importantísimo, y más lo es en el proceso de la elaboración, pues con ello la mejora de la priorización del gasto, está garantizada. Con la información del cumplimiento de indicadores de desempeño se alienta a las entidades ejecutoras a que los resultados que logren, harán que reciban mayores recursos.

Robinson \& Last (2009) señalan que existen una serie de requisitos para lograr una implementación del Presupuesto por Resultados exitosa, que pasan por elaborar indicadores de desempeño sencillos, una sólida base de información de resultados, una estructura programática para el presupuesto, una modificación de los sistemas de información financiera y contable, reducir los controles del presupuesto tradicional, además de la rigidez del gasto, y finalmente reconducir la estrategia de acción del Ministerio de Economía y Finanzas hacia el éxito de este nuevo sistema presupuestario.

Según el Ministerio de Economía y Finanzas \& Cooperación Alemana implementada por la GIZ (2016), “...el Presupuesto por Resultados es una estrategia de gestión pública que vincula la asignación de recursos a productos y resultados medibles a favor de la población..." (p.7), requiriéndose la presencia de una enunciación clara de los resultados a conquistar, el compromiso para alcanzarlos, quienes y que harán los responsables, los procedimientos para la generación de información de resultados, productos y de las herramientas de gestión institucional, y finalmente la rendición de cuentas. Mediante la Ley $\mathrm{N}^{\circ} 29142$, Ley del Presupuesto del año 2008, comienza la implementación del Presupuesto por Resultados con la aprobación de los primeros cinco Programas Presupuestales, número que ha ido 
incrementándose progresivamente, teniéndose al año 2015, ochenta y cinco (85) Programas Presupuestales.

En el Perú, el Presupuesto por Resultados, se implementa a través de cuatro instrumentos; i) Los programas presupuestales, que son los bienes y servicios que la Administración Pública va a entregar para lograr un resultado en favor de la población; ii) El Seguimiento, es el proceso de recolección y análisis de información de desempeño de los programas, la medición del cumplimiento de los objetivos; iii) Las Evaluaciones Independientes, que es analizar el diseño, la implementación, y la mejora de una intervención pública aterrizada en el programa y el impacto que se está logrando en el bienestar de la ciudadanía; iv) Incentivos a la Gestión, que trata de otorgar una serie de recursos financieros siempre y cuando se den resultados por mejoras en la gestión municipal básicamente.

\section{Materiales y métodos}

El diseño de investigación fue de tipo no experimental, longitudinal y correlacional predictivo. Para la realización del presente trabajo de investigación, se consideró como población al total de municipalidades o gobiernos locales de la región La Libertad al 2015, que según el Instituto Nacional de Estadística e Informática (INEI) y Transparencia Económica del Ministerio de Economía y Finanzas, totalizan 83. Por tratarse de una población poco numerosa en comparación con las más de 1800 municipalidades a nivel nacional, y considerando la relevancia del estudio para la Región La Libertad, se trabajó con el total de la población, se hizo un censo, es decir se analizó la gestión del presupuesto por resultados y la calidad del gasto en las 83 municipalidades o gobiernos locales de la Región La Libertad.

La Unidad de análisis estuvo constituida por las municipalidades o gobiernos locales de la Región la Libertad, en las que se analizó los cuatro instrumentos del presupuesto por resultados y como se vienen ejecutando en el Perú; de su análisis y comprensión integral se extrajeron conclusiones y se verificó su relación para y por la calidad del gasto en beneficio directo de la población. Estos cuatro instrumentos, objeto de análisis son: Los Programas Presupuestales donde analizaron los reportes de programas presupuestales del Ministerio de Economía y Finanzas del Perú (MEF) y Reportes de Avances y Consolidación del presupuesto por resultados en el Perú - MEF; para el segundo instrumento de Seguimiento, se analizaron los reportes del seguimiento de ejecución presupuestal por programas presupuestales, por fuente de recursos, por dominio geográfico, Reportes del Instituto nacional de estadística e informática (INEI) y el reporte de indicadores de Resulta-MEF; para el tercer instrumento de las Evaluaciones independientes se analizó el reporte de evaluaciones de diseño y ejecución presupuestal - MEF, y para el cuarto instrumento de las evaluaciones de Impacto (EI), y los Incentivos a la gestión se analizaron el reporte de Evaluaciones de Impacto realizados por el MEF y el Reporte de indicadores de Resulta-MEF.

\footnotetext{
"Visión de Futuro" Año 17, Volumen N²4 N², Julio - Diciembre 2020 - Pág. 37 - 59

URL de la Revista: http://visiondefuturo.fce.unam.edu.ar/index.php/visiondefuturo/index

URL del Documento: https://visiondefuturo.fce.unam.edu.ar/index.php/visiondefuturo/issue/view/18

ISSN 1668 - 8708 - Versión en Línea

E-mail: revistacientifica@,fce.unam.edu.ar
} 
Por parte del autor de la presente investigación se realizó un análisis con base en el modelo econométrico de diferencias en diferencias, tomando como base los reportes de cada instrumento del $\mathrm{PpR}$ ya mencionados anteriormente. Finalmente, también se analizaron reportes o comentarios de entrevistas a expertos, considerando las competencias de profesionales en el tema, para profundizar en la metodología en cuestión.

Con base en los objetivos del estudio y en la metodología planteada, resultó imprescindible basarnos en la banco de datos que proveen, en primero lugar del aplicativo del Ministerio de Economía y Finanzas del Perú (MEF) que realiza seguimiento al presupuesto por resultados, principalmente de los indicadores de desempeño de los programas presupuestales, por otro lado tenemos la información provista por el portal de Transparencia Económica - Consulta amigable del Ministerio de Economía y Finanzas del Perú (MEF), en dicho portal se gestiona una base de datos muy potente que realiza el seguimiento de la ejecución presupuestal, correspondiente a las Unidades Ejecutoras (UEs) del Gobierno Nacional, los Gobiernos Regionales y las municipalidades o los Gobiernos Locales; identificando plenamente la fuente y el rubro del financiamiento de los diferentes tipos de gastos y además de cómo se estructura el gasto. Dada la naturaleza de nuestro estudio fue necesario la consulta adicional de otras fuentes estadísticas del mismo corte, provenientes de diferentes entidades básicamente del INEI como encuestas realizadas por este organismo, tales como el ENAPRES (Encuesta Nacional de Programas Presupuestales), ENAHO (Encuesta Nacional de Hogares sobre Condiciones de Vida y Pobreza), ENDES (Encuesta Demográfica y de Salud Familiar), ENEDU (Encuesta Nacional a Instituciones Educativas ), entre otros.

En el desarrollo de la presente investigación se hizo uso tanto de la estadística descriptiva, mostrando tablas de distribución de frecuencias, porcentajes, gráficos, entre otros; asimismo la hipótesis formulada fue objeto de verificación, con el empleo de la estadística inferencial, para determinar la relación entre la variable independiente y la variable dependiente.

Se hizo uso del análisis de regresión múltiple, que es una técnica de análisis multivariable en el que se establecerá una relación funcional para la calidad del gasto y una serie de variables independientes o explicativas, que se definieron a partir de la descomposición del presupuesto por resultados; así se estimaron los coeficientes de regresión que determinaron el efecto que las variaciones de las variables independientes tienen sobre el comportamiento de la variable dependiente. La medida de la bondad del ajuste de la función estimada viene dada por el coeficiente de correlación múltiple, y el coeficiente de determinación, que es el cuadrado del anterior, expresa la proporción de la varianza de la variable dependiente explicada por el modelo de regresión (Montero, 2011).

Finalmente se utilizó la evaluación de impacto, donde se buscó determinar si la calidad 
del gasto cambió debido a alguna intervención de política pública, en este caso por efectos del presupuesto por resultados. Generalmente para determinar dicha causalidad potencial se requiere conocer el estado contrafactual de la intervención, para ello ésta investigación utilizó la estrategia econométrica basada en el estimador Differences in Differences (DD); para llegar a ello fue necesario establecer dos periodos marcados el antes y el después de la implementación de la gestión del presupuesto por resultados, y también dos tipos de grupos, a los que se denominaron tratados y control; determinando las características o criterios que hagan valedera esta posición de grupos. Con el establecimiento de todo ello y siguiendo la rigurosidad econométrica y estadística, el siguiente paso fue establecer la especificación del modelo, detallando la mejor estrategia para la obtención de resultados robustos. (Del Pozo, Guzmán y Pucarmayta, 2013).

\section{Resultados}

Respecto a la ejecución presupuestal, se analizó la eficiencia de la ejecución de todos los programas presupuestales implementados en los años de referencia, conforme se iban implementando (hay que recordar que el PpR, se ha implementado de forma gradual), se puede observar que, a nivel de La Libertad, región en estudio, el total del gasto acumulado desde el 2008 al 2015 es de S/. 10077 millones, con un PIM ${ }^{1}$ de S/. 12008 millones, lo que equivale a un casi $84 \%$ de nivel de ejecución o eficiencia, mientras que comparativamente a nivel de los 83 gobiernos Locales, el gasto acumulado del 2012 al 2015 es de S/. 2134 millones, mientras que el PIM es de S/. 3217 millones, lo que equivale a un $66 \%$ de nivel de ejecución o eficiencia. (Ver tablas $\mathrm{N}^{\circ} 1$ y N²).

Respecto a la ejecución presupuestal de los programas elegidos para el análisis econométrico y de regresión lineal múltiple, y que fueran seleccionados por su mayor incidencia en la región de estudio y a nivel de los Gobiernos Locales de la misma. El programa de reducción del costo, tiempo e inseguridad vial en el sistema de transporte terrestre logra el porcentaje de ejecución o eficiencia del gasto con $72.1 \%$, mientras que los programas nacionales de saneamiento urbano y rural alcanzan un $58.1 \%$ y $62.5 \%$ de eficiencia promedio, respectivamente. El programa de acceso y uso adecuado de los servicios públicos de telecomunicaciones e información, registra en promedio sólo un $58.1 \%$ de ejecución.

Por otro lado, el programa articulado nutricional registra un mayor nivel de ejecución en el 2014 con $83 \%$, sin embargo, su nivel de ejecución total es de $69.2 \%$, menor a la barrera ${ }^{2}$

\footnotetext{
1 PIM o Presupuesto institucional modificado: presupuesto actualizado de la entidad pública a consecuencia de las modificaciones presupuestarias, tanto a nivel institucional como a nivel funcional programático, efectuadas durante el año fiscal, a partir del PIA o Presupuesto inicial de apertura.

2 El Ministerio de Economía y Finanzas (MEF) ha establecido en los reportes de seguimiento a los Programas Presupuestales el valor de $75 \%$ como nivel referencial de ejecución anual de los Programas Presupuestales señalando que los programas que no alcancen dicho valor se encontrarían en riesgo alto de inadecuada ejecución anual.

"Visión de Futuro" Año 17, Volumen N²4 N², Julio - Diciembre 2020 - Pág. 37 - 59

URL de la Revista: http://visiondefuturo.fce.unam.edu.ar/index.php/visiondefuturo/index

URL del Documento: https://visiondefuturo.fce.unam.edu.ar/index.php/visiondefuturo/issue/view/18

ISSN 1668 - 8708 - Versión en Línea

E-mail: revistacientifica@,fce.unam.edu.ar
} 
del $75 \%$, lo que denota un riesgo alto de inadecuada ejecución anual, observable con mayor notoriedad en los años 2013 (47\%) y 2015 (59\%). El programa Logros de aprendizaje de estudiantes de EBR, sólo en el 2014, supera la barrera, llegando a 79\%, su promedio de ejecución es del $66.3 \%$. El promedio de ejecución global de los programas analizados en el presente estudio, es de $66 \%$, lo que pone en riesgo alto de inadecuada ejecución, a dichos programas, desde el 2012 al 2015. (Ver tabla $\left.N^{\circ} 3\right)$.

Tabla N 1. Ejecución de los Programas Presupuestales (PP) en La Libertad Periodo 2008 -2015 (En millones de S/.)

\begin{tabular}{|c|c|c|c|c|c|c|c|c|c|c|c|c|c|c|c|}
\hline \multirow{2}{*}{ Región } & \multicolumn{3}{|c|}{$\begin{array}{c}2008 \\
\mathrm{PP}^{3}: 05\end{array}$} & \multicolumn{3}{|c|}{$\begin{array}{c}2009 \\
\text { PP: } 09\end{array}$} & \multicolumn{3}{|c|}{$\begin{array}{c}2010 \\
\text { PP: } 15\end{array}$} & \multicolumn{3}{|c|}{$\begin{array}{c}2011 \\
\text { PP: } 24\end{array}$} & \multirow[t]{2}{*}{$\begin{array}{c}\text { PIM } \\
\text { Acumulado }\end{array}$} & \multirow[t]{2}{*}{$\begin{array}{c}\text { Gasto } \\
\text { Acumulado }\end{array}$} & \multirow[t]{2}{*}{$\%$} \\
\hline & $\mathrm{N}^{\circ} \mathrm{PP}^{4}$ & $\mathrm{PIM}^{5}$ & Gasto & $\begin{array}{l}\mathbf{N}^{\circ} \\
\mathrm{PP}\end{array}$ & PIM & Gasto & $\begin{array}{l}\mathrm{N}^{\circ} \\
\mathrm{PP}\end{array}$ & PIM & Gasto & $\begin{array}{l}\mathbf{N}^{\circ} \\
\mathrm{PP}\end{array}$ & PIM & Gasto & & & \\
\hline La Libertad & 5 & 250 & 214 & 9 & 219 & 182 & 12 & 480 & 388 & 20 & 697 & 549 & 1646 & 1333 & $81 \%$ \\
\hline \multirow{2}{*}{ Región } & & $\begin{array}{l}2012 \\
P P: 60\end{array}$ & & & $\begin{array}{r}2013 \\
\text { PP: } 6 \\
\end{array}$ & & & $\begin{array}{r}2014 \\
\text { PP: } 7\end{array}$ & & & $\begin{array}{c}2015 \\
P P: 85\end{array}$ & & \multirow{2}{*}{$\begin{array}{c}\text { PIM } \\
\text { Acumulado }\end{array}$} & $\begin{array}{c}\text { Gasto } \\
\text { Acumulado }\end{array}$ & \multirow[t]{2}{*}{$\%$} \\
\hline & $\mathrm{N}^{\circ} \mathrm{PP}$ & $\mathrm{PIM}$ & Gasto & $\begin{array}{l}\mathbf{N}^{\circ} \\
\mathbf{P P}\end{array}$ & PIM & Gasto & $\begin{array}{l}\mathbf{N}^{\circ} \\
\mathrm{PP}\end{array}$ & $\mathrm{PIM}$ & Gasto & $\begin{array}{l}\mathbf{N}^{\circ} \\
\mathrm{PP}\end{array}$ & PIM & Gasto & & & \\
\hline La Libertad & 38 & 1728 & 1403 & 46 & 2476 & 2039 & 54 & 2827 & 2393 & 64 & 3331 & 2909 & $362^{10}$ & 8744 & $84 \%$ \\
\hline \multicolumn{13}{|c|}{ TOTAL } & $0088^{12}$ & 10077 & $83.9 \%$ \\
\hline
\end{tabular}

Fuente: Transparencia Económica - MEF

Tabla $N^{\circ}$ 2. Ejecución de los Programas Presupuestales (PP) en los Gobiernos Locales de La Libertad Periodo 2012 -2015 (En millones de S/.)

\begin{tabular}{|c|c|c|c|c|c|c|c|c|c|c|c|c|c|c|c|}
\hline \multirow{3}{*}{ La Libertad } & \multicolumn{3}{|c|}{2012} & \multicolumn{3}{|c|}{2013} & \multicolumn{3}{|c|}{2014} & \multicolumn{3}{|c|}{2015} & \multirow{3}{*}{$\begin{array}{c}\text { PIM } \\
\text { Acumulado }\end{array}$} & \multirow{3}{*}{$\begin{array}{c}\text { Gasto } \\
\text { Acumulado }\end{array}$} & \multirow{3}{*}{$\%$} \\
\hline & \multicolumn{3}{|c|}{ PP: 38} & \multicolumn{3}{|c|}{ PP: 46} & \multicolumn{3}{|c|}{ PP: 54} & \multicolumn{3}{|c|}{ PP: 64} & & & \\
\hline & $\mathbf{N}^{\circ} \mathrm{PP}$ & PIM & Gasto & $\begin{array}{l}\mathrm{N}^{\circ} \\
\mathrm{PP}\end{array}$ & PIM & Gasto & $\begin{array}{l}\mathrm{N}^{\circ} \\
\mathrm{PP}\end{array}$ & PIM & Gasto & $\begin{array}{l}\mathbf{N}^{\circ} \\
\mathrm{PP}\end{array}$ & PIM & Gasto & & & \\
\hline $\begin{array}{c}\text { Gobiernos } \\
\text { Locales }\end{array}$ & 26 & 572 & 342 & 31 & 896 & 565 & 29 & 946 & 678 & 30 & 803 & 549 & 3217 & 2134 & $66 \%$ \\
\hline
\end{tabular}

Fuente: Transparencia Económica - MEF

\footnotetext{
"Visión de Futuro" Año 17, Volumen No 24 N², Julio - Diciembre 2020 - Pág. 37 - 59

URL de la Revista: http://visiondefuturo.fce.unam.edu.ar/index.php/visiondefuturo/index

URL del Documento: https://visiondefuturo.fce.unam.edu.ar/index.php/visiondefuturo/issue/view/18

ISSN 1668 - 8708 - Versión en Línea

E-mail: revistacientifica@,fce.unam.edu.ar
} 
Tabla N 3. Ejecución Según Programa Presupuestal en estudio, a Nivel De Los Gobiernos Locales de La Libertad Años 2012 - 2015 (Las cifras se expresan en miles de soles)

\begin{tabular}{|c|c|c|c|c|c|c|c|c|c|c|c|c|c|c|c|}
\hline \multirow{2}{*}{ Programa Presupuestal } & \multicolumn{3}{|c|}{2012} & \multicolumn{3}{|c|}{2013} & \multicolumn{3}{|c|}{2014} & \multicolumn{3}{|c|}{2015} & \multirow{2}{*}{$\begin{array}{l}\text { PIM } \\
\text { Acumu } \\
\text { l. }\end{array}$} & \multirow{2}{*}{$\begin{array}{c}\text { Gasto } \\
\text { Acumu } \\
\text { l. }\end{array}$} & \multirow{2}{*}{$\%$} \\
\hline & $\mathrm{PIM}$ & Gasto & $\%$ & $\mathrm{PIM}$ & Gasto & $\%$ & PIM & $\begin{array}{c}\text { Gast } \\
0\end{array}$ & $\%$ & PIM & Gasto & $\%$ & & & \\
\hline $\begin{array}{c}\text { Programa Articulado } \\
\text { Nutricional }\end{array}$ & 64 & 48 & $75 \%$ & 19 & 9 & $47 \%$ & 15 & 12 & $83 \%$ & 14 & 8 & $59 \%$ & 112 & 77 & $69.2 \%$ \\
\hline $\begin{array}{c}\text { Acceso y Uso Adecuado De } \\
\text { Los Servicios Públicos De } \\
\text { Telecomunicaciones E } \\
\text { Información } \\
\end{array}$ & 5 & 3 & $60 \%$ & 0.6 & 0.3 & $49 \%$ & 0.03 & 0.03 & $100 \%$ & 0.4 & 0.2 & $45 \%$ & 6 & 4 & $58.1 \%$ \\
\hline $\begin{array}{c}\text { Reducción Del Costo, } \\
\text { Tiempo E Inseguridad Vial En } \\
\text { El Sistema De Transporte } \\
\text { Terrestre } \\
\end{array}$ & 131 & 90 & $69 \%$ & 151 & 99 & $65 \%$ & 136 & 111 & $82 \%$ & 95 & 69 & $73 \%$ & 512 & 369 & $72.1 \%$ \\
\hline $\begin{array}{l}\text { Programa Nacional De } \\
\text { Saneamiento Urbano }\end{array}$ & 73 & 49 & $67 \%$ & 92 & 53 & $57 \%$ & 76 & 35 & $47 \%$ & 77 & 48 & $62 \%$ & 318 & 185 & $58.1 \%$ \\
\hline $\begin{array}{l}\text { Programa Nacional De } \\
\text { Saneamiento Rural }\end{array}$ & 68 & 36 & $53 \%$ & 155 & 92 & $60 \%$ & 193 & 129 & $67 \%$ & 149 & 96 & $64 \%$ & 565 & 353 & $62.5 \%$ \\
\hline $\begin{array}{l}\text { Logros De Aprendizaje De } \\
\text { Estudiantes De EBR }\end{array}$ & 123 & 65 & $53 \%$ & 190 & 109 & $58 \%$ & 176 & 138 & $79 \%$ & 146 & 108 & $74 \%$ & 635 & 421 & $66.3 \%$ \\
\hline TOTAL & 464 & 291 & $63 \%$ & 608 & 362 & $60 \%$ & 596 & 427 & $72 \%$ & 480 & 329 & $69 \%$ & 2148 & 1409 & $66 \%$ \\
\hline
\end{tabular}

Fuente: Transparencia Económica - MEF

\section{Evaluación de impacto: modelo diferencias en diferencias (DID)}

Se busca determinar en primer lugar si el nivel de ejecución financiera en la función de salud, cambió debido a alguna intervención de política pública, en este caso por efectos del presupuesto por resultados y el establecimiento de las denominadas categorías presupuestales o programas presupuestales.

Generalmente para determinar dicha causalidad potencial se requiere conocer el estado contrafactual de la intervención, para ello esta investigación se ha favorecido de lo desarrollado por Del Pozo, Guzmán y Pucarmayta (2013), quienes, en su estudio para la estimación del impacto de los ingresos por canon minero en las variables de interés utiliza la estrategia econométrica basada en el estimador Differences in Differences (DiD).

De acuerdo con lo revisado anteriormente y con lo referido por el Ministerio de Economía y Finanzas, el presupuesto por resultados se inició en el 2007 bajo el liderazgo de la DGPP (Dirección General del Presupuesto Público) y busca fortalecer el vínculo entre los recursos asignados y los resultados obtenidos a favor de la población. En 2010 se crea la Dirección de Calidad del Gasto Público, una de las cinco direcciones de línea de la Dirección General de Presupuesto Público (DGPP), con el objetivo de impulsar, mediante instrumentos y metodologías, la Reforma del Presupuesto por Resultados (PpR); teniendo como funciones principales la conducción del sistema de seguimiento y evaluación del desempeño del gasto público.

Las consideraciones metodológicas para definir el periodo óptimo de evaluación del progreso del presupuesto por resultado, implica un largo proceso para obtener y generar información. Este proceso consta de tres momentos; el primero, consiste en determinar la

\footnotetext{
“Visión de Futuro" Año 17, Volumen N²4 N², Julio - Diciembre 2020 - Pág. 37 - 59

URL de la Revista: http://visiondefuturo.fce.unam.edu.ar/index.php/visiondefuturo/index

URL del Documento: https://visiondefuturo.fce.unam.edu.ar/index.php/visiondefuturo/issue/view/18

ISSN 1668 - 8708 - Versión en Línea

E-mail: revistacientifica@,fce.unam.edu.ar
} 
fuente de información más apropiada para cuantificar el valor de cada uno de los indicadores generados; luego, elaborar el algoritmo de cálculo (sintaxis de programación) que permite obtener el valor estimado del indicador para determinado periodo temporal. En el tercer momento, se realiza una evaluación del progreso de los indicadores, en la cual se desarrolla el cálculo del indicador, así como el de otros datos estadísticos como su desviación estándar, coeficiente de variación e intervalos de confianza. Por otro lado, a nivel de Gobiernos Locales, sólo se asignó recursos por S/. 158 millones en el 2008, mientras que en el 2009 y 2010, no recibieron recurso alguno.

Por lo cual considerando todo ello, se han establecido dos periodos marcados de evolución, el primero hasta 2009 (periodo ex ante), y el segundo desde 2011, periodo de mediciones constantes de los indicadores del presupuesto por resultados. Para la implementación del estimador DiD la estrategia de identificación implica definir dos tipos diferentes de distritos, los distritos tratados y los distritos control. Para este estudio se tuvieron en cuenta varios criterios que nos dieran la mejor elección del grupo de tratamiento y del grupo control. El primer criterio es el índice de desarrollo humano (IDH) a nivel distrital, en la región La Libertad, cuya clasificación refiere un IDH alto, para valores de 0.80 y 1 ; un IDH medio, para valores de 0.50 a 0.799; y un IDH Bajo de 0 a 0.499 . Con este criterio, tenemos a 12 distritos de los 83 que conforman la región La Libertad, que tienen un IDH mayor a 0.50, agrupándolos en el grupo de distritos control.

El otro criterio que se tuvo en cuenta es la clasificación que realiza la Dirección de Calidad del Gasto público, y que utiliza en el instrumento del Presupuesto por resultados del Programa de Incentivos a la mejora de la Gestión Municipal, donde agrupa a las distritos en cuatro clasificaciones: Municipalidades Tipo A, (Distritos principales en una determinada región), Municipalidades Tipo B; Municipalidades que No son Ciudades Principales y que tiene más de 500 VVUU (unidades de vivienda), y por ultimo las Municipalidades que No son Ciudades Principales y que tiene menos de 500 VVUU (unidades de vivienda); confirmando con ello a los distritos tratados con el anterior criterio; quedando con ello definidos 12 distritos control y 71 distritos tratados.

\footnotetext{
"Visión de Futuro" Año 17, Volumen No 24 N², Julio - Diciembre 2020 - Pág. 37 - 59

URL de la Revista: http://visiondefuturo.fce.unam.edu.ar/index.php/visiondefuturo/index

URL del Documento: https://visiondefuturo.fce.unam.edu.ar/index.php/visiondefuturo/issue/view/18

ISSN 1668 - 8708 - Versión en Línea

E-mail: revistacientifica@,fce.unam.edu.ar
} 
Tabla N4. Distritos según IDH que son Grupo Control (GC)

\begin{tabular}{lcc}
\hline DISTRITO & IDH & GRUPO \\
\hline Magdalena De Cao & 0.5022 & GC \\
\hline Huanchaco & 0.5050 & GC \\
\hline La Esperanza & 0.5119 & GC \\
\hline Moche & 0.5153 & GC \\
\hline Laredo & 0.5197 & GC \\
\hline San Pedro De Lloc & 0.5212 & GC \\
\hline Santiago De Cao & 0.5256 & GC \\
\hline Pacasmayo & 0.5302 & GC \\
\hline Casa Grande & 0.5388 & GC \\
\hline Chocope & 0.5438 & GC \\
\hline Trujillo & 0.6694 & GC \\
\hline Victor Larco Herrera & 0.7248 & GC
\end{tabular}

Fuente: PNUD³- Perú

GxPit $=\beta(0$ it $)+\beta($ tratamiento $i)+\beta($ periodo $t)+\beta(D i D$ (tratamiento*periodo $) i t)+d i+a t+\mu i t$

Con base en la metodología y los criterios especificados anteriormente, siguiendo la rigurosidad econométrica y estadística, a continuación, presentamos la especificación del modelo econométrico, detallando la mejor estrategia para la obtención de resultados robustos. Empleando una especificación lineal de probabilidad, la regresión para estimar el impacto de la distribución con la nueva metodología del presupuesto por resultados, en el gasto por programa presupuestal, quedaría definida de la siguiente manera:

Dónde: GxPit es la variable dependiente gasto por programa presupuestal, $\beta(0$ it) es la constante; $\beta$ (tratamiento i) es una variable dummy que toma el valor 1 , si el distrito pertenece al grupo de tratamiento, definidas según los criterios antes descritos; $\beta$ (periodo $t$ ) es una variable dummy que toma el valor 1 para los años 2011, 2012, 2013, 2014 y 2015 (después de la política), y 0 para los años 2005, 2006, 2007, 2008, 2009 (antes de la política), $\beta$ (DiD (tratamiento*periodo)it) es el estimador del diff and diff, que resulta de operar tratamiento*periodo; di son efectos fijos ${ }^{4}$ a nivel Distrito; at son efectos fijos de años; y $\mu$ it es el término de error. Se trabajará con este modelo el Programa Articulado Nutricional. El modelo empírico planteado incorpora algunas interacciones relevantes para lograr el objetivo del presente estudio en este sentido, en particular el parámetro $\beta$ (DiD it) captura la interacción para los distritos con mayores ingresos destinados según la nueva metodología del

\footnotetext{
${ }^{3}$ Programa de las Naciones Unidas para el Desarrollo. ecuación presentada, cuando los mismos tendrían algún grado de endogeneidad.

"Visión de Futuro" Año 17, Volumen N² 24 N², Julio - Diciembre 2020 - Pág. 37 - 59

URL de la Revista: http://visiondefuturo.fce.unam.edu.ar/index.php/visiondefuturo/index

URL del Documento: https://visiondefuturo.fce.unam.edu.ar/index.php/visiondefuturo/issue/view/18

ISSN 1668 - 8708 - Versión en Línea

E-mail: revistacientifica@fce.unam.edu.ar
}

4 Según, Montero (2011), es decir suponeque el error ( $\varepsilon i t)$ puede descomponerse en dos una parte fija, constante para cada distrito (ri) y otra aleatoria que cumple los requisitos MCO ( $\mu \mathrm{it})$, o sea ( $(\varepsilon i \mathrm{t}=\mathrm{ri}+\mu \mathrm{it}$ ); lo que es equivalente a obtener una tendencia general por regresión dando a cada individuo un punto de origen (ordenadas) distinto; así lo mismo para at a nivel de año. El modelo de Efectos Fijos permite obtener estimaciones consistentes de los efectos marginales de los regresores considerados en la 
presupuesto por resultados, antes y después del periodo de corte, definido previamente, con esto se busca estimar el impacto directo del presupuesto por resultados sobre el gasto por programa presupuestal.

Según Del Pozo, Guzmán y Pucarmayta (2013) el supuesto clave del estimador DiD implica que los factores inobservables que determinan la exposición al tratamiento son constantes en el tiempo. En las ecuaciones planteadas, las dummies de tiempo dan cuenta de cambios en el tiempo de las medidas de gasto por programa, en los distritos expuestos a tal presupuesto, los efectos fijos di y at dan cuenta de características que se asumen invariantes en el tiempo. Por otro lado, para Montero (2011), hay que tener en cuenta que en las estimaciones por DiD existe un problema potencial de correlación serial, sobretodo, en lo referente a la variable dependiente (GxPit) la cual estaría correlacionada de forma positiva y donde la variable tratamiento o exposición a alguna política pública cambia muy poco dentro de la unidad de tratamiento o exposición a través del tiempo, por lo cual es necesario corregir los errores estándar, por clúster y heterocedasticidad a nivel de distrito, dicha corrección por clúster de los errores estándar permite dar cuenta de la potencial correlación espacial de distritos expuestas a shocks y condiciones de variables similares. ${ }^{5}$

En la tabla $\mathrm{N}^{\circ} 5$ se presentan los resultados del impacto de la nueva política del presupuesto por resultados en el gasto ejecutado en el programa articulado nutricional, con base en estimaciones por differences in differences; dichos resultados provienen de las estimaciones de la ecuación inicial planteada. Ésta se hizo primero con una especificación lineal-lineal, y luego con la especificación donde la variable dependiente es logaritmo y las independientes lineales; en ellas encontramos primero, la estimación por mínimos cuadrados ordinarios y luego se incluyen efectos fijos a nivel de distritos y años; en ambos casos los errores son corregidos por heterocedasticidad y por clúster a nivel de distritos. El coeficiente DiD obtenido para la primera especificación es un valor positivo, que denota una relación positiva con el gasto por programa, es decir dicho coeficiente explica el incremento en el gasto por programa (articulado nutricional) de los distritos de la Región La Libertad, producto de la nueva política del presupuesto por resultados; lo cual resulta razonable considerando la prioridad asignada a los programas presupuestales, uno de los cuatro instrumentos del PpR en el Perú. En la segunda especificación, los coeficientes están recogiendo la variación porcentual $^{6}$ del gasto por programa que se experimentó producto de la implementación de la política, es decir en el caso de $\mathrm{MCO}$, el programa articulado nutricional tuvo un incremento porcentual de $895.8 \%$ (este resultado se obtiene realizando la aplicación matemática de elevar la constante matemática e al coeficiente de DiD del modelo MCO y restándole la unidad, para

\footnotetext{
5 En cada regresión de MCO efectuada en el programa econométrico Stata, se le adicionará la función robust cluster (distritos), para conseguir tal fin.

${ }^{6}$ Para obtener dicha variación porcentual, hay que realizar esta operación matemática: Variación \% = $\left(e^{\beta_{\text {DiD }}}-1\right)^{*} 100$

"Visión de Futuro" Año 17, Volumen $N^{\circ} 24$ N², Julio - Diciembre 2020 - Pág. 37 - 59

URL de la Revista: http://visiondefuturo.fce.unam.edu.ar/index.php/visiondefuturo/index

URL del Documento: https://visiondefuturo.fce.unam.edu.ar/index.php/visiondefuturo/issue/view/18

ISSN 1668 - 8708 - Versión en Línea

E-mail: revistacientifica@fce.unam.edu.ar
} 
a dicho resultado multiplicarlo por 100, obteniendo una variación producto de la implementación del $\mathrm{PpR}$ ), sin duda un incremento notablemente superior al escenario ex ante de la implementación del presupuesto por resultados como política de estado.

Tabla $\mathbf{N}^{\circ}$ 5. Resultados del modelo para el programa articulado nutricional

\begin{tabular}{c|c|c|c|c}
\hline \multirow{2}{*}{ Modelo } & \multicolumn{2}{|c|}{ LIN-LIN } & \multicolumn{2}{c}{ LOG-LIN } \\
\cline { 2 - 5 } & MCO & EF & MCO & -0.3759286 \\
& -132977.1 & 29591.27 & -1.704311 & $(1.17317)$ \\
\hline \multirow{2}{*}{ Tratamiento } & $(137002.1)$ & $(72196.62)$ & $(1.206483)$ & -0.7967583 \\
& -77711.12 & -224131.2 & -1.591794 & $(0.7612062)$ \\
\hline \multirow{2}{*}{ Periodo } & $(105819.7)$ & $(128933.4)^{*}$ & $(0.8424084)^{*}$ & 1.851401 \\
& 278479.5 & 278479.5 & 2.298412 & $(1.173151)$ \\
\hline \multirow{2}{*}{ DiD } & $(116295.7)^{\star *}$ & $(123034)^{* *}$ & $(1.074127)^{* *}$ & 10.97675 \\
& 148817.7 & 87241.86 & 11.93932 & $(0.7045595)^{* * *}$ \\
\hline \multirow{2}{*}{ Constante } & $(136318)$ & $(72749.29)$ & $(1.016592)^{\star * *}$ & 287 \\
\hline
\end{tabular}

Notas: $\mathrm{MCO}=$ Mínimos cuadrados ordinarios; $\mathrm{EF}=$ efectos fijos; errores estándar robustos a heterocedasticidad.

Significancia al $1 \%\left({ }^{* * *}\right)$; significancia al $5 \%\left({ }^{* *}\right)$ y significancia al $10 \%\left({ }^{*}\right)$

Fuente: Estimaciones propias en STATA

\section{Análisis de la gestión del presupuesto por resultados y la calidad del gasto con modelo de regresión múltiple}

Como se sabe, el modelo de regresión múltiple se utiliza para predecir el comportamiento de una determinada variable, es decir la variable dependiente o criterio, en función de otras variables, las denominadas independientes o explicativas o también regresores o predictores. Para la presente investigación se desarrolla un análisis de regresión múltiple a nivel de todas las regiones del Perú, por tener la información disponible de los indicadores de resultados de los programas presupuestales a nivel de regiones; configurándose en un escenario comparativo y de discusión sobre la relación del presupuesto por resultados en indicadores claves, que vistos en ese campo de acción son de igual extensivos a la parte provincial y distrital de las regiones, y en este caso, además de la comparativa con la estadística descriptiva a nivel de la Región La Libertad.

$\mathrm{Y}=$ Presupuesto Ejecutado En Programas Presupuestales (PExP) es la variable dependiente.

El modelo quedaría definido en:

$P E X P=\beta 0+\beta 1 X 1+\beta 2 X 2+\beta 3 X 3+\beta 4 X 4+\beta 5 X 5+\beta 6 X 6+\beta 7 X 7+\beta 8 X 8+\beta 9 X 9+\beta 10 X 10+\beta 11 X 11+\beta 12$ $X 12+\beta 13 \times 13+\beta 14 \times 14+\beta 15 \times 15+\beta 16 \times 16+\mu$ it (2)

Donde:

$\beta 0=$ constante $;$ y $\beta 1=\beta 2=\ldots . \beta n$ son todos los coeficientes.

$\mathrm{X} 1=$ Eficiencia \% (Devengado/Pim)

\footnotetext{
"Visión de Futuro" Año 17, Volumen No 24 N², Julio - Diciembre 2020 - Pág. 37 - 59

URL de la Revista: http://visiondefuturo.fce.unam.edu.ar/index.php/visiondefuturo/index

URL del Documento: https://visiondefuturo.fce.unam.edu.ar/index.php/visiondefuturo/issue/view/18

ISSN 1668 - 8708 - Versión en Línea

E-mail: revistacientifica@fce.unam.edu.ar
} 
x2= Prevalencia De Desnutricion Cronica En Menores De 5 Años (\%)

X3= Tiempo Promedio De Traslado Con Medios De Transporte Motorizado A La Municipalidad

Distrital (Minuto)

X4= Cobertura De Alcantarillado U Otras Formas De Disposición De Excretas-Urbano (\%)

X5= Cobertura De Agua Por Red Pública-Urbano (\%)

X6= Cobertura De Alcantarillado U Otras Formas De Disposición De Excretas-Rural (\%)

X7= Cobertura De Agua Por Red Pública-Rural (\%)

X8= Proporción De Pobl. Con Acceso A Internet (\%)

X9= Proporción De Pobl. Con Cobertura De Telef Móvil (\%)

X10 $=\%$ De Estudiantes De 2do De Primaria En Nivel Satisfactorio Comp Lectora

X11 $=$ \% De Estudiantes De 2do De Primaria En Nivel Satisfactorio Matemática

X12= Índice De Desarrollo Humano (Idh)

X13= Esperanza De Vida Al Nacer (Años)

X14= Población Con Educ. Secundaria Completa (\%)

X15= Años De Educación (Poblac. 25 Y Más) (Años)

X16= Ingreso Familiar Per Cápita (Nivel Salarial X Mes)

$\mu \mathrm{it}=$ Término de error

Formulación de la hipótesis estadística:

Ho: $\beta 1=\beta 2=\cdots \beta p=0$

H1: $\exists \boldsymbol{\beta} \mathbf{j} \neq \mathbf{0}$ (Al menos uno de los coeficientes es distinto de cero)

Según los resultados de las tablas $\mathrm{N}^{\circ} 6,7$ y 8 , se rechaza la Hipótesis nula, y se acepta la hipótesis alterna, por lo tanto, la ecuación de regresión múltiple queda confirmada en:

$P E x P=1980029009+318968173 X 1+506564319 \times 2+406553 \times 3+522760323 X 4-$ $881227 X 5-413811771 X 6+123398620 X 7-463147424 X 8+27108709 X 9+801369869$ X10 -872532343 X11 + 4475613797 X12 - 40561384 X13 - 2989162 X14 - 62305581 X15 $1316348 \times 16+\mu$ it

El nivel de significación global, considerando un nivel de confianza del 5\%, y contrastándolo a partir del valor del Prob (F Statistic), que para el modelo especificado corresponde a 0.0000 , vemos que es menor al 0.05 de nivel de significancia, lo que estaría significando que el modelo globalmente considerado resulta significativo, lo que implica que tiene la virtualidad de explicar el comportamiento de la variable dependiente, en este caso el presupuesto ejecutado en programas presupuestales.

Existe una relación significativa entre las variables en estudio, la cual se contrasta con 
el coeficiente de correlación de 0.69. La bondad del ajuste del modelo especificado se contrasta a partir del estadístico R-Squared que corresponde a: 0,4824, lo cual quiere decir, que es explicativo en un casi $50 \%$, en otras palabras, la relación entre las 16 variables independientes del modelo especificado, dan cuenta en un mediano porcentaje del comportamiento de la variable dependiente, en este caso el presupuesto ejecutado en programas presupuestales.

Se puede observar relaciones directas y significativas con las variables independientes de: Cobertura De Alcantarillado U Otras Formas De Disposición De Excretas-Urbano (\%); Cobertura De Alcantarillado U Otras Formas De Disposición De Excretas-Rural (\%); Proporción De Pobl. Con Acceso A Internet (\%); \% De Estudiantes De 2do De Primaria En Nivel Satisfactorio Comp. Lectora; \% De Estudiantes De 2do De Primaria En Nivel Satisfactorio Matemática; Esperanza De Vida Al Nacer (Años); lo cual denota a priori calidad del gasto por la nueva metodología del presupuesto por resultados, en los programas de Saneamiento Urbano y Rural; Programa de Acceso y Uso adecuado de Servicios de Telecomunicaciones, y en el Programa Logros de Aprendizaje de EBR.

Tabla $\mathbf{N}^{\circ} 6$. Análisis de varianza

\begin{tabular}{cccccc}
\hline & $\begin{array}{c}\text { Grados } \\
\text { de } \\
\text { libertad }\end{array}$ & $\begin{array}{c}\text { Suma de } \\
\text { cuadrados }\end{array}$ & $\begin{array}{c}\text { Promedio de } \\
\text { los cuadrados }\end{array}$ & F & $\begin{array}{c}\text { Valor crítico de } \\
\text { F }\end{array}$ \\
\hline Regresión & 16 & $1.89346 \mathrm{E}+18$ & $1.18341 \mathrm{E}+17$ & 4.83458554 & 0.00000 \\
\hline Residuos & 83 & $2.03168 \mathrm{E}+18$ & $2.44781 \mathrm{E}+16$ & & \\
\hline Total & 99 & $3.92514 \mathrm{E}+18$ & & & \\
\hline
\end{tabular}

Fuente: Estimaciones propias en STATA

Tabla $\mathbf{N}^{\circ}$ 7. Estadísticas de la regresión

\begin{tabular}{lr}
\hline Coeficiente de correlación múltiple & 0.6945 \\
\hline Coeficiente de determinación $\mathrm{R}^{\wedge} 2$ & 0.4824 \\
\hline $\mathrm{R}^{\wedge} 2$ ajustado & 0.3826 \\
\hline Error típico & 156454690 \\
\hline Observaciones & 100 \\
\hline
\end{tabular}

Fuente: Estimaciones propias en STATA

\footnotetext{
"Visión de Futuro" Año 17, Volumen N² 24 N², Julio - Diciembre 2020 - Pág. 37 - 59

URL de la Revista: http://visiondefuturo.fce.unam.edu.ar/index.php/visiondefuturo/index

URL del Documento: https://visiondefuturo.fce.unam.edu.ar/index.php/visiondefuturo/issue/view/18

ISSN 1668 - 8708 - Versión en Línea

E-mail: revistacientifica@,fce.unam.edu.ar
} 
Tabla $N^{\circ} 8$. Resultados del modelo de regresión múltiple

\begin{tabular}{|c|c|}
\hline Variables & $\begin{array}{l}\text { Modelo de regresión lineal múltiple (LIN-LIN } \\
\text { con MCO) }\end{array}$ \\
\hline Eficiencia \% (Devengado/PIM) & $318968173(317203969)$ \\
\hline $\begin{array}{c}\text { Prevalencia De Desnutrición Crónica En Menores De } 5 \\
\text { Años }(\%)\end{array}$ & $506564319(400092783)$ \\
\hline $\begin{array}{c}\text { Tiempo Promedio De Traslado Con Medios De } \\
\text { Transporte Motorizado A La Municipalidad Distrital } \\
\text { (Minuto) }\end{array}$ & $406553(409726)$ \\
\hline $\begin{array}{c}\text { Cobertura De Alcantarillado U Otras Formas De } \\
\text { Disposición De Excretas-Urbano (\%) }\end{array}$ & $522760323(1908772233)^{\star * *}$ \\
\hline Cobertura De Agua Por Red Pública-Urbano (\%) & $-881227(2400765)$ \\
\hline $\begin{array}{c}\text { Cobertura De Alcantarillado U Otras Formas De } \\
\text { Disposición De Excretas-Rural (\%) }\end{array}$ & $-413811771(2,33011927)^{*}$ \\
\hline Cobertura De Agua Por Red Pública-Rural (\%) & $123398620(110988873)$ \\
\hline Proporción De Robl. Con Acceso A Internet (\%) & $-463147424(249850204)^{\star}$ \\
\hline Proporción De Robl. Con Cobertura De Jelef Móvil (\%) & 27108709 (130492493) \\
\hline $\begin{array}{l}\text { \% De Estudiantes De 2do De Primaria En Nivel } \\
\text { Satisfactorio Comp Lectora }\end{array}$ & $801369869(469113174)^{\star}$ \\
\hline $\begin{array}{c}\text { \% De Estudiantes De 2do De Primaria En Nivel } \\
\text { Satisfactorio Matemática }\end{array}$ & $-872532343(520866428)^{\star}$ \\
\hline Índice De Desarrollo Humano (IDH) & $4475613797(3220952277)$ \\
\hline Esperanza De Vida AI Nacer (Años) & $-40561384(12288596)^{\star \star \star *}$ \\
\hline Población Con Educ. Secundaria Completa (\%) & $-2989162(3943289)$ \\
\hline Años De Educación (Roblaç 25 Y Más) (Años) & $-62305581(62573638)$ \\
\hline Ingreso Familiar Per Cápita (Nivel Salarial X Mes) & $-1316348(869869)$ \\
\hline Constante & $1980029009(770008275)^{\star *}$ \\
\hline
\end{tabular}

Notas: $\mathrm{MCO}=$ mínimos cuadrados ordinarios; errores estándar robustos a heterocedasticidad

Significancia al $1 \%\left(^{(* *}\right)$; significancia al $5 \%\left({ }^{* *}\right)$; significancia al $10 \%\left(^{*}\right)$

Fuente: Estimaciones propias en STATA

\section{CONCLUSIÓN}

El diagnóstico de la gestión del presupuesto por Resultados arroja un nivel de ejecución o eficiencia a nivel de La Región La Libertad, no guarda relación con lo referido por Raymundo (2011), quien resalta que se espera que el rol presupuestal, sea un rol más activo; ya que el gasto público debe estar necesariamente vinculado a los objetivos de desarrollo que están priorizados en los planes de gobierno; y si bien se observa que hay mecanismos que ayudan a éste propósito, el nivel de ejecución por debajo de la franja establecida, denota un nivel que da pie a no concretización de resultados e impactos esperados de largo alcance y de largo plazo.

Para Ausejo (2010) el Presupuesto por Resultados debe contemplar una serie de principios y técnicas para diseñar, ejecutar, hacer seguimiento y evaluar, el conjunto de intervenciones que tienen dos lineamientos claros; primeros los bienes y servicios que se

\footnotetext{
"Visión de Futuro" Año 17, Volumen N² 24 N², Julio - Diciembre 2020 - Pág. 37 - 59

URL de la Revista: http://visiondefuturo.fce.unam.edu.ar/index.php/visiondefuturo/index

URL del Documento: https://visiondefuturo.fce.unam.edu.ar/index.php/visiondefuturo/issue/view/18

ISSN 1668 - 8708 - Versión en Línea

E-mail: revistacientifica@,fce.unam.edu.ar
} 
brindan a la población objetivo, que son los productos; y segundo los cambios que se generarían en el bienestar que son los resultados, y que tienen que concretarse con eficiencia, eficacia y equidad, algo que vemos no viene ocurriendo, conforme los resultados presentados, para los gobiernos locales de La Libertad.

Según Prieto (2012) los actuales indicadores de evaluación presupuestal no consideran los beneficios sociales que se debe brindar a la población, e incluso son desconocidos por quienes elaboran dicha evaluación. Los indicadores miden solamente el monto del gasto, pero no la calidad del mismo. La toma de decisiones con respecto al gasto público no es muy eficiente, ya que generalmente el gasto se centra en los gastos de capital y no en gastos que mejoren las condiciones de vida de la población.

En términos de cobertura se ha logrado un avance significativo en la implementación de los principales instrumentos de la reforma, pero su incidencia para orientar el proceso presupuestario hacia un enfoque por resultados es limitada. Los programas presupuestales, y el seguimiento de su desempeño, presentan deficiencias en su implementación y no cuentan con mecanismos efectivos para utilizar de manera sistemática la información de desempeño en el proceso presupuestario.

Se midió la calidad del gasto, identificando los avances en otros instrumentos del presupuesto por Resultados, así con respecto a la evaluación de impacto del presupuesto por resultados en el gasto ejecutado en el programa articulado nutricional, con base en estimaciones por differences in differences; el coeficiente DiD obtenido es un valor positivo, que denota una relación positiva o directa con el gasto por programa, es decir dicho coeficiente explica el incremento en el gasto por programa de los distritos de la Región La Libertad, producto de la nueva política del presupuesto por resultados; lo cual resulta razonable considerando la prioridad asignada a los programas presupuestales, uno de los cuatro instrumentos del PpR en el Perú.

Existe una relación directa y significativa entre la gestión del presupuesto por resultados y la calidad del gasto; lo cual se comprueba en primer lugar con el nivel de significancia global del modelo estadístico [Prob (F Statistic) $=0.0000$ ], la cual, además se contrasta con el coeficiente de correlación de 0.69 . Se observan relaciones directas y significativas con las variables de Cobertura De Alcantarillado U Otras Formas De Disposición De Excretas-Urbano (\%); Cobertura De Alcantarillado U Otras Formas De Disposición De Excretas-Rural (\%); Proporción De Pobl. Con Acceso A Internet (\%); \% De Estudiantes De 2do De Primaria En Nivel Satisfactorio Comp. Lectora; \% De Estudiantes De 2do De Primaria En Nivel Satisfactorio Matemática; Esperanza De Vida Al Nacer (Años); lo cual denota que la gestión del Presupuesto por resultados si se relaciona significativamente con la calidad del gasto en los programas de Saneamiento Urbano y Rural; Programa de Acceso y Uso adecuado de

\footnotetext{
"Visión de Futuro" Año 17, Volumen N²4 N², Julio - Diciembre 2020 - Pág. 37 - 59

URL de la Revista: http://visiondefuturo.fce.unam.edu.ar/index.php/visiondefuturo/index

URL del Documento: https://visiondefuturo.fce.unam.edu.ar/index.php/visiondefuturo/issue/view/18

ISSN 1668 - 8708 - Versión en Línea

E-mail: revistacientifica@,fce.unam.edu.ar
} 
Servicios de Telecomunicaciones, y en el Programa Logros de Aprendizaje de EBR, básicamente.

Estos resultados hallan eco con los hallados por Mejía (2014) quien obtiene que el Presupuesto por Resultados como herramienta si influye en la mejora de la Gestión Gerencial de un gobierno local que dicho investigador analizó, porque parte de una visión integrada de planificación y articulación que busca acciones articuladas en función a la resolución de los problemas críticos que afectan a la población, los mismos que se han comprobado en este estudio.

Nuestros resultados también concuerdan con lo hallado por Prieto (2012) quien refiere que la aplicación del Presupuesto por resultados en las municipalidades del Perú mejora la calidad del gasto público ya que ellos son destinados a favorecer los niveles de vida de la población. La manera como mejorar la calidad de vida de la población es mediante la asignación de recursos presupuestales en los programas estratégicos tales como: Mejorar la desnutrición crónica infantil, Mejorar la calidad de la educación básica, o Mejorar la dación de servicios básicos a la población, aspectos que han sido demostrados con solvencia en la presente investigación.

\section{REFERENCIAS}

Armijo, M. y Espada, M. (2014). Calidad del gasto público y reformas institucionales en América Latina. CEPAL - Serie Macroeconómica del Desarrollo, 156, 1-82. http://hdl.handle.net/11362/37012

Ausejo, F. (2010). Descentralización y Presupuesto por Resultados. Diplomado sobre gestión por resultados. Consorcio de Investigación Económica y Social. http://www.gestionporresultados.cies.org.pe/sites/default/files/modulo-iii-ppr.pdf

Carrasco, M. (2015). Presupuesto por Resultados: una perspectiva comparativa para la experiencia peruana. Conferencia El presupuesto por Resultados en el Perú, Lima, Perú.

http://sgp.pcm.gob.pe/wpcontent/uploads/2015/10/GABRIELA CARRASCO MEF.pdf

Del Pozo, C., Guzmán, E y Pucarmayta, V. (2013). ¿Minería y bienestar en el Perú?: evaluación de impacto del esquema actual (ex-post) y esquemas alternativos (ex-ante) de re-distribución del canon minero, elementos para el debate. CIES consorcio de investigación económica y social. https://www.cies.org.pe/es/investigaciones/medioambiente-recursos-naturales-y-energia/mineria-y-bienestar-en-el-peru

Franciskovic, J. (2013). Retos de la gestión pública: presupuesto por resultados y rendición de cuentas. Journal of Economics, Finance and Administrative Science, 18, 28-32. https://doi.org/10.1016/S2077-1886(13)70028-3

\footnotetext{
"Visión de Futuro" Año 17, Volumen N² 24 N², Julio - Diciembre 2020 - Pág. 37 - 59

URL de la Revista: http://visiondefuturo.fce.unam.edu.ar/index.php/visiondefuturo/index

URL del Documento: https://visiondefuturo.fce.unam.edu.ar/index.php/visiondefuturo/issue/view/18

ISSN 1668 - 8708 - Versión en Línea

E-mail: revistacientifica@,fce.unam.edu.ar
} 
Mejía, A. (2014). El presupuesto por resultados como herramienta para mejorar la Gestión Gerencial de la Municipalidad provincial de Barranca. [Tesis de pregrado, Universidad Nacional José Faustino Sánchez Carrión]. Lima, Perú. Repositorio UNJFSC. https://es.scribd.com/document/363437653/EL-PRESUPUESTO-POR-RESULTADOSCOMO-HERRAMIENTA-PARA-MEJORAR-LA-GESTION-GERENCIAL-pdf

Ministerio de Economía y Finanzas \& Cooperación Alemana implementada por la GIZ (2016). Programas Presupuestales. Diseño, Revisión y Articulación Territorial 2016. https://www.mef.gob.pe/contenidos/presu_publ/ppr/directiva_PP2016.pdf

Montero, R. (2011). Efectos fijos o aleatorios: test de especificación. Documentos de Discusión en Economía Aplicada. https://www.ugr.es/ montero/matematicas/especificacion.pdf

Prieto, M. (2012). Influencia De La Gestión Del Presupuesto Por Resultados En La Calidad Del Gasto En Las Municipalidades Del Perú (2006-2010) “Caso: Lima, Junín Y Ancash”. [Tesis de Doctorado, Universidad de San Martín de Porres]. Lima, Perú. Repositorio Académico USMP. http://www.repositorioacademico.usmp.edu.pe/handle/usmp/586 Raymundo, R. (2011). La Eficiencia En La Gestión Pública: El Caso De La Gestión De Inversión Pública Local En El Perú. ESGP Escuela de gestión pública. http://repositorio.ucss.edu.pe/handle/UCSS/93

Robinson, M. y Last, D. (2009). Un modelo básico de presupuestación por resultados. Notas técnicas y manuales sobre gestión financiera pública. https://blog-pfm.imf.org/files/fadtechnical-manual-1_spanish-translation.pdf

Schröder, P. (2013). Nueva Gestión Pública: Aportes para el buen gobierno. Oficina Regional América Latina. http://relial.org/uploads/biblioteca/44dbee76837e79a6c07bb8219d021843.pdf

Tanaka, E. (2011). Influencia del presupuesto por resultados en la Gestión Financiera Presupuestal del Ministerio de Salud. [Tesis de Maestría, Universidad Nacional Mayor de San Marcos]. Lima, Perú. CYBERTESIS Repositorio de Tesis Digitales. http://cybertesis.unmsm.edu.pe/handle/cybertesis/860

\section{REFERENCIAS BIBLIOGRÁFICAS}

\section{Vargas Merino, Jorge Alberto}

Maestro en Economía Universitat de Barcelona, España y Maestro en Administración de Negocios Universidad Nacional de Trujillo, Perú. Afiliación actual: Universidad Norbert Wiener, Facultad de Ingeniería y Negocios, Lima, Perú. Cargo: Docente de Investigación. Intereses actuales: Políticas públicas, Administración, Marketing, Economía, Innovación Social. 


\section{Zavaleta Chávez, Walter Enrique}

Posdoctorado en Ética, Responsabilidad Social y Derechos Humanos por la Universitat Abat Oliba, Barcelona, España; Doctor en Educación, Magíster en Docencia Universitaria, Magíster en Administración de Negocios y Relaciones Internacionales, Universidad César Vallejo de Trujillo, Perú. Afiliación actual: Universidad César Vallejo, Facultad de Ciencias Empresariales, Lima, Perú.

Cargo: Coordinador de la Escuela de Marketing y Dirección de Empresas Lima Norte. Intereses actuales: Responsabilidad Social, Administración, Marketing, Economía, Innovación Social, Derechos Humanos 